\title{
Evaluation de la sécurité des remblais en cours de construction: le code UDAM
}

\section{The assessment of the safety of earth dams during construction: the program UDAM}

\author{
A. NANDA (1) \\ CERMES $*$ \\ P. DELAGE \\ CERMES $^{*}$ \\ N. NEDJAT \\ Thésard à $\mathrm{EDF} / \mathrm{CNEH}^{* *}$ \\ J.-J. FRY \\ Ingénieur $\mathrm{EDF} / \mathrm{CNEH}^{* *}$ \\ B. GATMIRI \\ CERMES*
}

Rev. Franç. Géotech. n 62, pp. 23-34 (janvier 1993)

\section{Résumé}

La recrudescence des ruptures par glissement des remblais argileux au cours de la dernière décennie, en France (Mirgenbach, Mondely) ou à l'étranger (Carsington, Açu) a montré la difficulté de maîtriser la montée des pressions interstitielles en cours de construction au sein de ce type de barrage. Un code de calcul, UDAM, a été développé en vue de prévoir la génération de pression interstitielle et de quantifier la sécurité du remblai. Son originalité réside dans la prise en compte du caractère polyphasique du sol. Les pressions d'air, pressions d'eau et les déplacements sont les inconnues nodales, le coefficient de sécurité est défini pour chaque élément. Le comportement non saturé est basé sur la notion de surfaces d'état, mise en forme par Alonso. Après la présentation des principales hypothèses du code, quelques applications sont présentées.

\section{Abstract}

The increasing number of failures of clayey fills by sliding during the last decade in France (Mirgenbach, Mondely) or abroad (Carsington, Açu) showed the problems of controlling the increase of pore pressure during construction in this kind of dam. A computer code, UDAM, has been developped to forecast the pore pressure and evaluate the safety of the fill. The main originality is the triphasic approach of the behaviour of the soil. Air pressure, water pressure and displacements are the nodal unknown variables, the local safety factor is calculated at each element. The unsaturated constitutive equations are derived from the state surfaces model elaborated by Alonso. Following the presentation of the main assumptions made in the program, some applications are demonstrated.

- Ecole Nationale des Ponts et Chaussées, 93167 Noisy-le-Grand Cedex.

* 73373 Le Bourget-du-Lac Cedex.

(1) Adresse actuelle: D-167 Defence Colony, New Delhi-110024, Inde. 


\section{PATHOLOGIE DE LA CONSTRUCTION DES REMBLAIS ARGILEUX}

Le 20 août 1982, un ouvrier remarquait une fissure longitudinale sur le parement amont du barrage de Mirgenbach. Il appela son chef de chantier, et tous les deux constatèrent que les deux lèvres continuaient à s'écarter. Elles s'écartèrent pendant plus de 15 jours, créant une marche de $4 \mathrm{~m}$ (fig. 1). Ils venaient de mettre en évidence le paroxysme de la rupture du talus amont, amorcée en fait depuis plus de deux mois. L'auscultation du talus aval révéla des vitesses de tassement de $2 \mathrm{~cm} /$ jour qui s'accélérèrent irrérnédiablement le 13 septembre. Victimes des mêmes causes, les parties centrales du parement amont et aval glissèrent et déplacèrent $30000 \mathrm{~m}^{3}$. La digue de Mirgenbach était un barrage homogène en argile de $22 \mathrm{~m}$ de hauteur. Ce type d'ouvrage, couramment réalisé, est faussement banal. L'incident de Mirgenbach souligne trois difficultés majeures:

- l'estimation de la génération de la pression de l'eau au sein de l'argile;

- le contrôle de l'argile mise en place;

- le suivi et linterprétation des pressions d'eau par l'auscultation.

En effet, le glissement démarra au contact de deux ou trois couches trop humides dont l'eau piégée monta en pression au cours du chargement. $\mathrm{Ni}$ le contrôle de l'humidité, ni l'interprétation de l'auscultation ne surent évaluer à sa juste valeur le risque d'instabilité.
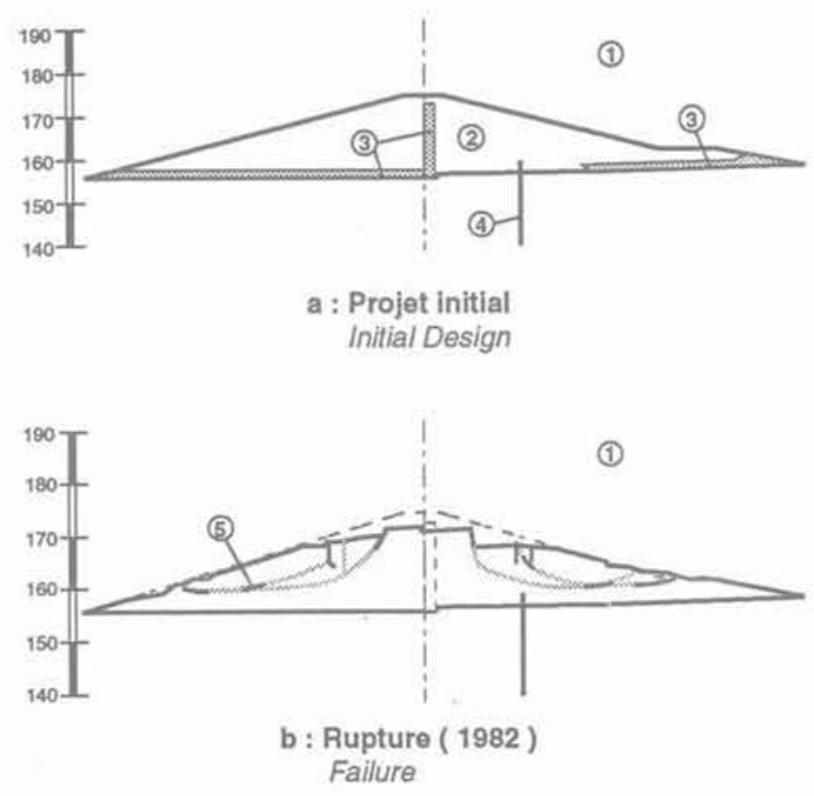

$\begin{array}{ll}\text { (1) Amont } & \text { (1) Upstream } \\ \text { (2) Argile } & \text { (2) Clay } \\ \text { (3) Filtre ot drain } & \text { (3) Filter and drain } \\ \text { (4) Paroi mouldo } & \text { (4) Cut off wall } \\ \text { (5) Surfaces de glissement } & \text { (5) Slip surfaces }\end{array}$

Fig. 1. - Glissement du barrage de Mirgenbach. Fig. 1. - Failure of the Mirgenbach Dam.
Cette lacune n'est pas inhérente au cas de ce chantier. A la même époque, d'autres ouvrages subirent des glissements de nature identique. A Mondely, un glissement apparut au cours de la première vidange en mai 1981. L'analyse rétrospective mit en évidence que le glissement traversait une zone humide, compactée lors d'une saison pluvieuse, qui généra des pressions importantes. En 1984, la rupture de Carsington, en Grande-Bretagne, s'explique non seulement par la faiblesse de la résistance de l'argile de fondation, mais aussi par les fortes pressions au sein du noyau. Enfin, G. Post relate qu'en 1981, le barrage d'Açu, au Brésil, a subi un glissernent provoqué par des pressions importantes.

L'origine commune à la rupture de ces ouvrages est l'apparition de fortes pressions d'eau, conséquente à la mise en place d'une argile dotée d'une humidité forte et d'une perméabilité faible. Le glissement provoqué par la génération de pression est certainement la principale pathologie des remblais en terre construits actuellement (les ruptures de vidange rapide sont signalées en France au cours de la premiêre moitié du siècle). La survivance de cette pathologie s'explique en partie par la rusticité des approches utilisées. Il manque un cadre théorique qui relie la nature du sol et les dispositifs constructifs à l'évaluation de la sécurité. Une telle approche alarmerait mieux l'ingénieur de chantier des risques qu'il encourt.

\section{PRESSIONS D'EAU ET D'AIR ET SUCCION DANS UN BARRAGE}

Avant d'aborder les méthodes d'évaluation de la pression d'eau au sein d'un remblai, il n'est pas inutile d'en rappeler les principales manifestations. Tombées en désuétude, ces dernières avaient été bien observées par LAMBE (1961) et BISHOP (1964).

\subsection{La succion}

Après répandage d'une couche, l'argile contient de l'air entre ses mottes. Le compactage réduit le volume de l'air en le chassant. Ainsi la pression de l'air $\mathrm{P}$ est proche de la pression atmosphérique. La pression d'eau $\mathrm{P}_{w}$ est par contre inférieure à la pression atmosphérique. La différence P.P $\mathrm{P}_{w}$ a été appelée succion par les hydrologues. Cetté succion est à l'origine de la remontée capillaire, mise en évidence par la loi de Jurin, à travers les interstices du sol. En l'absence d'effets osmotiques, elle est la pression capillaire reliée à la courbure des ménisques par la loi de Laplace (fig. 2):

$$
P_{\mathrm{a}} \cdot \mathrm{P}_{\mathrm{w}}=A\left(\frac{1}{\mathrm{r}^{1}}+\frac{1}{\mathrm{r}^{2}}\right)
$$

avec: A, tension superficielle de l'eau

$r_{1}$ et $r_{2}$, rayons de courbure principaux du mé. nisque.

Il est à noter que succion est employée souvent lorsque l'air est à la pression atmosphérique. En revanche lorsque la pression d'eau est positive, lair est soit chassé, s'il est en phase continue, et la succion devient nulle, soit occlu, donc discontinu, et la succion n'est autre que la pression capillaire au sein des bulles cap. tives. 


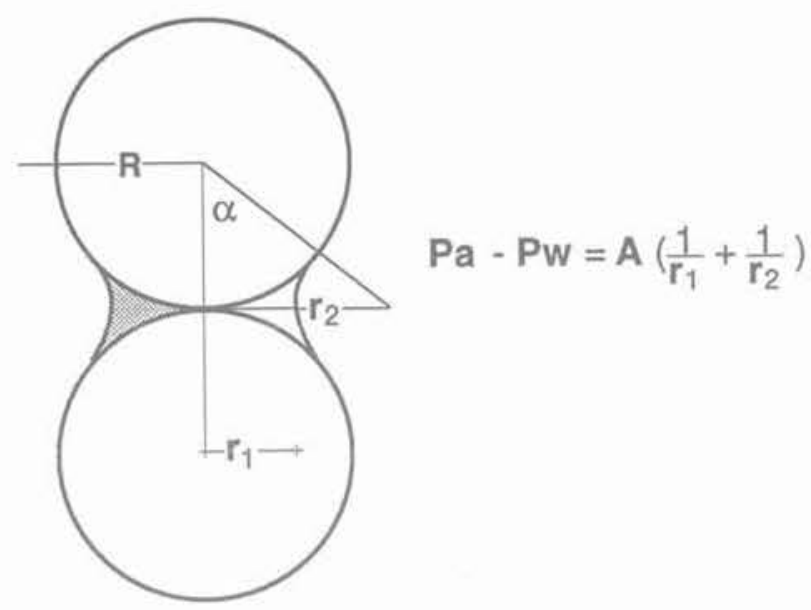

Fig. 2. - Loi de Laplace,

Fig. 2. Laplace law.

\subsection{Variation de la succion initiale avec le compactage}

Pour distinguer les trois pressions présentes dans les interstices du sol, nous préférerons employer le terme de pression d'eau plutôt que celui de pression inters. titielle.

La capillarité, qui n'est autre que l'attraction des molécules et des ions à la surface des particules sur les molécules d'eau, augmente fortement avec la surface spécifique. Ainsi à énergie de compactage équivalente, la succion initiale augmente avec la plasticité du sol. Le tableau 1 regroupe quelques résultats de succions initiales, à l'Optimum Proctor Normal, mesurées en labo. ratoire. La succion ne dépasse $50 \mathrm{kPa}$ pour un limon ou un sable peu argileux, avoisine $200 \mathrm{kPa}$ pour une argile moyennement plastique et dépasse $300 \mathrm{kPa}$ pour une argile très plastique.

La succion initiale diminue fortement quand la teneur en eau de compactage augmente. En effet, lattraction superficielle des grains ne se fait pratiquement plus ressentir sur les dernières couches de molécules d'eau qui entourent le grain. Plus généralement, la succion diminue quand le degré de saturation augmente. La relation qui lie la succion à la densité et à la teneur est complexe. Elle dépend de la structure et du chemin suivi, comme la montré LAMBE (1961). Pour cette raison, il importe de la mesurer sur des échantillons intacts.

La figure 3 représente la variation de la succion avec la teneur en eau après compactage de l'argile de Mirgenbach. Cette relation fournit un moyen indirect de connaitre la teneur en eau de compactage, à partir des pressions mesurées par les cellules à cordes vibrantes du système d'auscultation. A Mirgenbach, la cellule B2 a donné une pression négative initiale de l'ordre de $20 \mathrm{kPa}$ qui correspondrait ici à une teneur en eau élevée, de l'ordre de $26 \%$ ou $w_{\mathrm{OPN}}+4 \%$, supérieure aux spécifications.

En gardant en mémoire les réserves d'usage sur la généralisation d'un résultat de laboratoire aux phénomènes in situ et sur le domaine d'utilisation de telles cellules limité, comme la tensiométrie, à une valeur de $80 \mathrm{kPa}$, on en déduit que de telles mesures de succion peuvent constituer un critère de contrôle de compactage à ne pas ignorer.

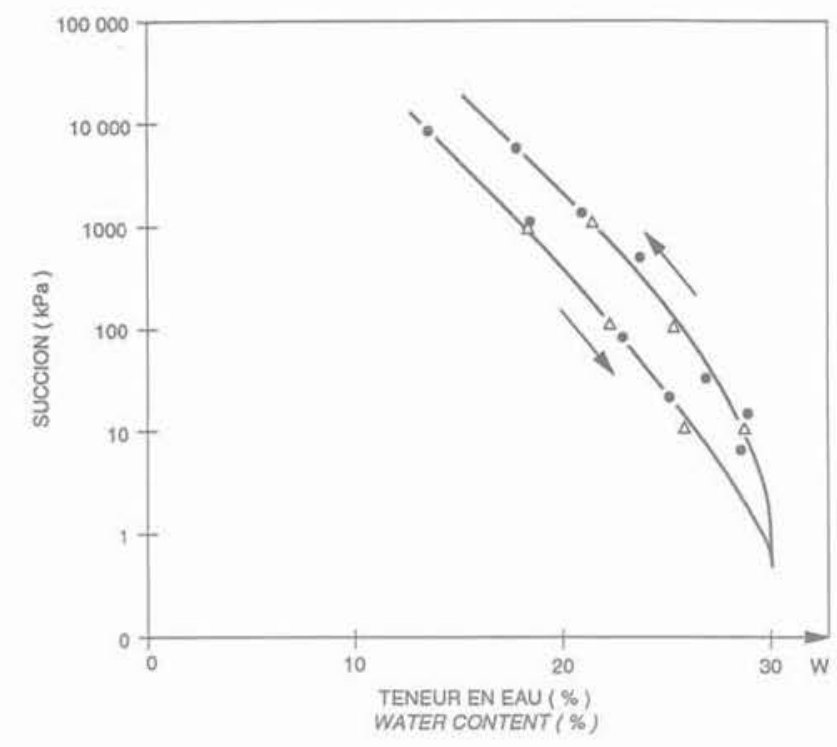

Fig. 3. - Succion en fonction de la teneur en eau : argile de Mirgenbach.

Fig. 3. - Suction as a function of water content: Mirgenbach clay.

Tableau 1. - Succion de certains sols compactés à I'OPN.

Table 1. - Suction of several compacted soils at the Optimum Standard Proctor.

\begin{tabular}{|c|c|c|c|c|c|c|}
\hline Sol & $\begin{array}{c}\text { Succion } \\
(\mathrm{kPa})\end{array}$ & $W_{\text {OPN }}(\%)$ & $\%<2 \mu$ & $I_{p}(\%)$ & $w_{L}(\%)$ & Auteur \\
\hline $\begin{array}{l}\text { Grave du Chambon } \\
\text { Limon de Lalybont } \\
\text { Sable argileux la Verne } \\
\text { Grave du Clou } \\
\text { Limon de Jossigny } \\
\text { Argile sableuse } \\
\text { Argile de Mangla } \\
\text { Argile de Selset } \\
\text { Argile brune }\end{array}$ & $\begin{array}{r}20 \\
30 \\
30 \\
50 \\
150 \\
170 \\
270 \\
350 \\
300\end{array}$ & $\begin{array}{l}9,7 \\
9,5 \\
18 \\
7,0 \\
18 \\
17,4 \\
16,2 \\
11,2 \\
29,0\end{array}$ & $\begin{array}{r}4 \\
4 \\
3 \\
6 \\
35 \\
26 \\
21 \\
20 \\
33\end{array}$ & $\begin{array}{l}4,6 \\
17 \\
2 \\
18 \\
24 \\
16,6 \\
16,6 \\
43\end{array}$ & $\begin{array}{l}25,6 \\
36 \\
23 \\
35 \\
40 \\
38,2 \\
33,1 \\
68\end{array}$ & $\begin{array}{l}\text { Fleureau } \\
\text { Bishop } \\
\text { Fleureau } \\
\text { Fleureau } \\
\text { Delage } \\
\text { USBR } \\
\text { Bishop } \\
\text { Bishop } \\
\text { Morris }\end{array}$ \\
\hline
\end{tabular}


Cette analyse rétrospective rappelle que la succion, ou la pression d'eau négative mesurée sur l'ouvrage, est un élément théorique important. (Une amélioration des mesures de succion in situ serait ainsi un apport intéressant dans le domaine de l'auscultation).

\subsection{Pression d'eau en cours de construction, exemple de Mirgenbach}

Au cours de la construction, le sol est comprimé, le volume d'air réduit et la pression d'air accrue, avec dissipation fonction de la perméabilité à l'air et de la distance à la surface, le degré de saturation augmente et la succion diminue. La figure 4 (BISHOP, DONALD and BLIGHT, 1961) montre l'évolution des pressions d'eau et d'air mesurées sur différents sols compactés, quand la contrainte est augmentée à l'appareil triaxial. Pour les échantillons de Selset $\left(w_{\mathrm{OPN}}+0,5 \%\right)$ et MAN. GLA ( $\left.w_{\mathrm{opN}}+2 \%\right)$, on observe qu'à partir d'une succion initiale proche de $140 \mathrm{kPa}$, on obtient lors de la compression égalité des pressions d'air et d'eau, pour des valeurs de contrainte de $700 \mathrm{kPa}$ et $420 \mathrm{kPa}$ res. pectivement.

On observe sur la plupart des barrages en argile que le niveau de contrainte n'est pas suffisant pour dissoudre tout le volume d'air contenu et l'argile reste non saturée. Dans une argile compactée à l'optimum, il est nécessaire d'appliquer une contrepression d'eau de $800 \mathrm{kPa}$ pour atteindre la saturation. Avec un degré de satura. tion initial plus fort de l'ordre de $95 \%$, une pression de $250 \mathrm{kPa}$ suffit.

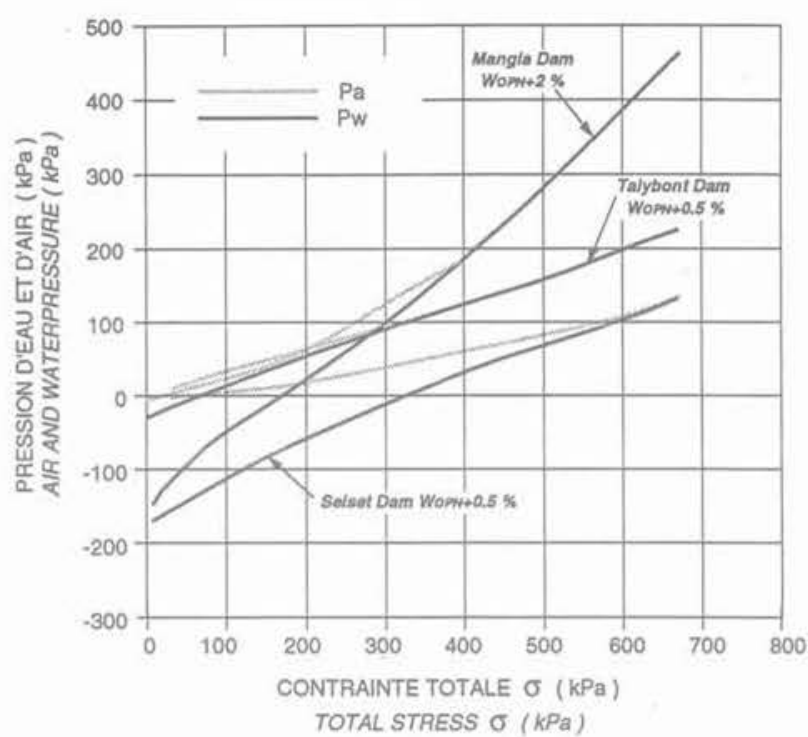

Fig. 4. - Pressions mesurées par BISHOP, DONALD et BLIGHT (1961).

Fig 4. - Pressures measured by BISHOP. DONALD and BLIGHT (1961).

Classiquement, seule la pression d'eau retient l'attention de l'ingénieur. En effet elle explique la plupart des cas extrêmes entraînant la pathologie. La figure 5 montre ainsi l'évolution de la pression d'eau mesurée par des cellules Télémac en deux endroits du barrage de Mirgenbach. La pression est reportée en fonction de la

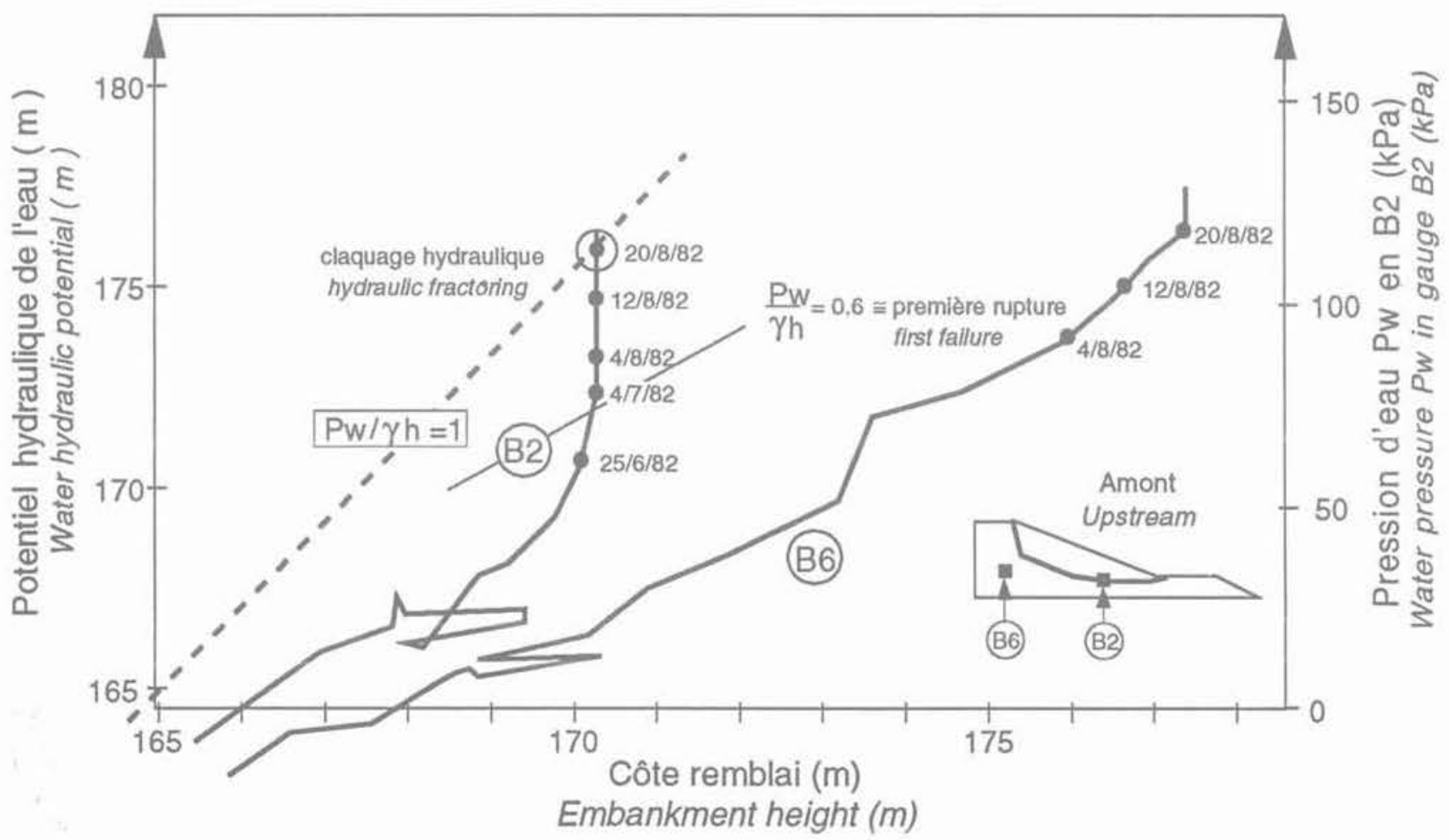

Fig. 5. - Pressions d'eau mesurées dans le barrage de Mirgenbach lors de la construction.

Fig. 5. - Water pressures measured in the Mirgenbach Dam during construction. 
montée du remblai. Les échelles ont été choisies pour que la pente de la droite passant par un point quelconque et l'origine soit égale au rapport de la pression d'eau à la contrainte totale verticale due au poids du remblai. On constate que ce rapport atteignit 1 au paroxysme du glissement. La rupture par glissement s'est accompagnée d'un claquage hydraulique local. Cela eut pour conséquence l'effondrement de la cohésion non drainée, le report de charge sur les parties adjacentes, la modification de l'écoulement et l'amplification de la rupture progressive.

La simulation de tels phénomènes n'est pas aisée. D'une part, des hypothèses simplifiées risquent d'aboutir à des résultats exagérément pessimistes: ainsi l'approche saturée est insuffisante pour expliquer le bon comportement des petits barrages en terre. D'autre part, la non prise en compte du couplage hydraulique-mécanique, des transferts de charge et des phases transitoires des calculs de stabilité actuels risque d'occulter les phénomènes les plus critiques et les plus dimensionnants.

Actuellement, des méthodes simplifiées sont traditionnellement employées pour évaluer les pressions et la stabilité en cours de construction. Comme nous allons le montrer, leur avantage réside dans la simplicité et l'économie de l'approche, leur inconvénient se résume à leur approximation.

\section{LES MÉTHODES TRADITIONNELLES DE PRÉVISION DES PRESSIONS}

Historiquement, les premières approches, les méthodes de BISHOP et de HILF, considèrent que le sol est non drainé. Cela est vérifié pour les argiles à plasticité moyenne ou forte, mais est trop pessimiste pour les argiles latéritiques, les limons et autres sols de perméabilité supérieure à $10^{-8} \mathrm{~m} / \mathrm{s}$. Aussi, les développements postérieurs intègrent la consolidation, dans une méthode découplée, comme celle de BISHOP (1957), ou dans une approche globale empirique, la méthode du coefficient ru.

\subsection{La méthode de BISHOP et les coefficients de SKEMPTON}

La première approche proposée par BISHOP consiste à mesurer au triaxial les coefficients $\mathrm{A}$ et $\mathrm{B}$ de Skempton :

$$
\mathrm{dP}_{\mathrm{w}}=\mathrm{B}\left(\mathrm{d} \sigma_{3}+\mathrm{A}\left(\mathrm{d} \sigma_{1}-\mathrm{d} \sigma_{3}\right)\right)
$$

où l'augmentation de pression d'eau, $\mathrm{dP}_{w}$ (souvent dénommée du), est provoquée d'une part par l'augmentation de contrainte totale de confinement isotrope, $\mathrm{d} \sigma_{3}$, et d'autre part par l'augmentation du déviateur, $\mathrm{d} \sigma_{1}-$ $\mathrm{d} \sigma_{3}$. Ces coefficients sont alors appliqués en tout point du barrage dont l'état de contrainte est calculé. La pression obtenu $\mathrm{P}_{\mathrm{w}}$ est alors rapportée à la containte principale majeure $\sigma_{1}$, pour obtenir le coefficient $\mathrm{B}$, plus pratique à introduire dans le calcul de stabilité par la méthode des tranches:

$$
\bar{B}=d P_{w} / d \sigma_{1}
$$

BISHOP (1957) a ainsi montré que dans la majorité des cas, la ruture est vraisemblable, si le coefficient $\bar{B}$ dépasse $60 \%$, exception faite des très petits barrages. Pour assurer un coefficient de sécurité de 1,3 ou 1,5 en fin de construction, une valeur de $50 \%$ ou $40 \%$ respectivement ne doit pas être dépassée en règle générale. En pratique, deux adaptations ont été dévelop. pées:

a) Tout d'abord l'expression de Henkel est préférée pour déterminer la pression sous l'état de contrainte tridimensionnel :

$$
\mathrm{dP}_{\mathrm{w}}=\mathrm{a} . \mathrm{I}+\mathrm{b} \cdot \mathrm{J}
$$

avec: I: $1^{\circ}$ invariant du tenseur de containte $\left(\sigma_{1}+\sigma_{2}+\sigma_{3}\right) / 3$

$$
J: 2^{\circ} \text { invariant }\left(\Sigma\left(\sigma_{i}-\sigma_{i}\right)^{2}\right)^{0.5}
$$

Avec l'hypothèse de déformation plane, souvent justifiée, la pression d'eau au sein du remblai se déduit de l'expression (2) en changeant le coefficient $A$ par $A_{d p}$ :

$$
A_{d p}=1 / 2+\sqrt{3}(3 A-1) / 6
$$

b) Ensuite le coefficient ru a été préféré à la place de $\mathrm{B} ;$ ru est le rapport de l'augmentation de pression d'eau sur la contrainte fictive de la colonne de sol à l'aplomb du point considéré :

$$
P_{w}=P_{w 0}+r u .(\gamma \cdot h)
$$

avec: $P_{w}$, pression d'eau sous $h$ mètres de remblai, $\mathrm{P}_{u 0}^{w}$, pression d'eau après compactage, $\gamma$, poids volumique humide du remblai.

Il était en effet judicieux d'utiliser ru en hypothèse, car ce paramètre est mesurable, donc vérifiable. D'autre part, moyennant l'hypothèse d'un chemin œdométrique, il fournit la pression en fonction des coefficients de Skempton et du rapport des contraintes principales $\mathrm{K}$ :

$$
\mathrm{K}=\mathrm{d} \sigma_{1} / \mathrm{d} \sigma_{3}
$$

avec: $\mathrm{d} \sigma_{1}$, l'incrément de contrainte principale majeure $\mathrm{d} \sigma_{3}$, l'incrément de contrainte principale mineure.

PELLS (1973) montre que pour un chargement supérieur au compactage, $\mathrm{K}$ n'est autre que le coefficient de poussée des terres au repos $\mathrm{K}_{\mathrm{o}}$ et ru prend l'expression suivante (identique à l'expression de $\mathrm{B}$ donnée par BISHOP (1954) dans le cas où $\sigma_{1}$ est verticale):

$$
r u=\frac{\left.B \cdot K_{o}+A\left(1-K_{o}\right)\right]}{1-B(1-A)\left(1-K_{o}\right)}
$$

Il apparaît ainsi que la génération de pression est prin. cipalement dépendante de B.

Les coefficients A et B sont représentatifs, s'ils sont me. surés sur des éprouvettes in situ et en nombre suffisant. Un minimum de 12 éprouvettes triaxiales est souhaitable, car l'hétérogénéité est importante. La figure 6 montre la variabilité de $\mathrm{B}$ en fonction du degré de sa. turation, constatée sur l'argile de Mirgenbach.

Souvent une corrélation reliant $B$ au degré de saturation est utilisée. Cette corrélation est incomplète. On pourrait 


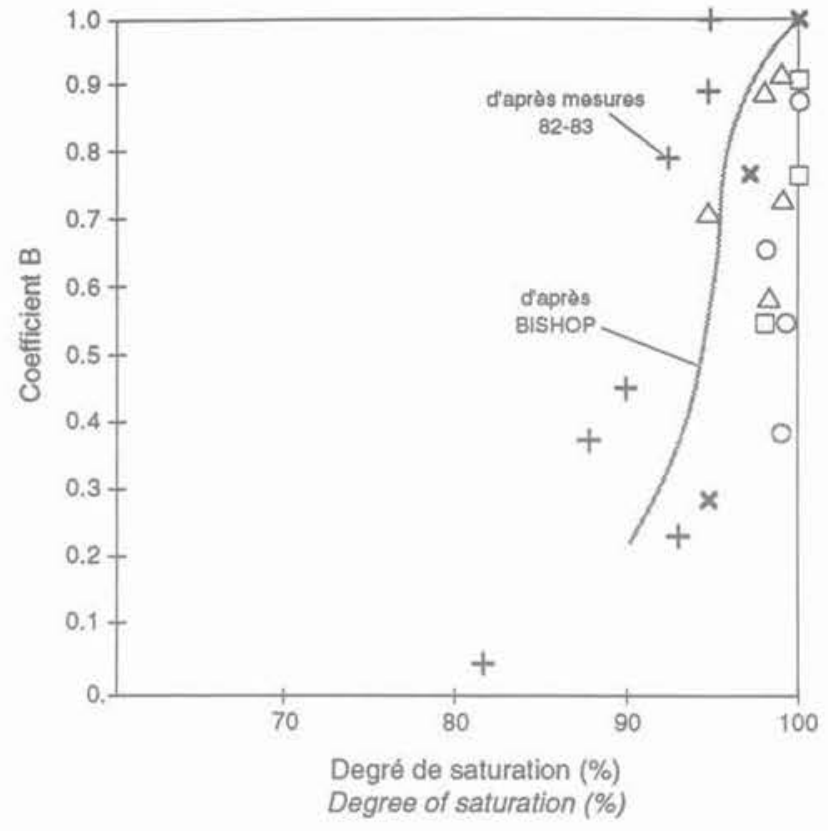

Fig. 6. - Valeurs du coefficient B de Skempton, fonction du degré de saturation mesurées sur l'argile du barrage de Mirgenbach.

Fig. 6. - Coefficient of Skempton B values, as a function of degree of saturation. measured on the Mirgenbach dam clay.

croire que la surpression d'eau augmente avec la densité après compactage, à teneur en eau identique. En fait, la pression dépend surtout de la teneur en eau initiale. Cela est confirmé par les corrélations obtenues sur la cohésion non drainée $c_{u}$ de l'argile compactée du barrage de Vieux Pré.

$$
2 c_{u}=1,93-0,217 w+0,05 \mathrm{~S}_{1}
$$

avec: un coefficient de corrélation R: 0,74 et 84 données, et $\mathrm{c}_{\mathrm{y}}$ en $10^{5} \mathrm{~Pa}$,

$w$ et $S$ sont respectivement la teneur en eau et le degré de saturation en $\%$.

Or en théorie, la génération de pression diminue avec la compressibilité, qui est inversement proportionnelle à la contrainte de compactage. Ainsi, contrairement à une opinion trop répandue, le compactage ne nuit pas à la stabilité. En revanche, un cisaillement violent et répété, tel celui créé par le trafic de chantier, risque de laminer et de feuilleter l'argile. Ainsi, un grand nombre de passes de compacteur sur une argile trop humide, non seulement n'augmente pas la densité, mais aussi cisaille largile. Il importe de dissocier ces deux phénomènes.

\subsection{La méthode de HILF}

Le paramètre $\mathrm{B}$ traduit en fait le rapport de la rigidité de la phase fluide sur la rigidité de l'ensemble:

$$
B=\left(E_{i} / n\right) /\left(E_{s}+E_{f} / n\right)
$$

avec: $\mathrm{E}$, module de la phase solide,

$\mathrm{E}_{\mathrm{f}}$, module de la phase fluide: eau + air, n, porosité.

HILF (1948) a exprimé directement la compressibilité du fluide en combinant la loi de Mariotte pour la compression de l'air et la loi de Henri pour la solubilité de l'air dans l'eau. Il en extrait la pression d'air au sein du sol compacté après chargement:

$$
\mathrm{Pa}_{1}=\mathrm{Pa}_{0} \cdot \mathrm{dv} /(\mathrm{Va}+\mathrm{HVw}-\mathrm{dv})
$$

avec: $\mathrm{Pa}_{1}$, pression d'air après chargement, assimilée à la pression d'eau,

$\mathrm{Pa}_{0}$. pression d'air après compactage, proche de la pression atmosphérique, dv, variation de volume du squelette,

Va, volume d'air après compactage,

$\mathrm{H}$, constante de Henry $=0,02$ à $16^{\circ}$ (variant de 0,022 à 0,018 entre 12 et $22^{\circ}$ ),

$\mathrm{HVw}$, volume d'air dissous.

La compressibilité du sol n'est pas exprimée par l'intermédiaire du module. Elle est extraite des essais œedométriques. La détermination de la pression d'air en est améliorée à condition que les essais soient non remaniés. La pression d'eau est assimilée à la pression d'air. Cela augmente la sécurité. Mais il est dommage, qu'après avoir fait allusion à la succion, HILF n'y prête plus attention. Un calcul, pas à pas, réactualisant la succion en fonction du degré de saturation testé sur les données de Mirgenbach, donne des résultats concor. dants à lauscultation (Tableau 2).

\subsection{Méthode empirique basée sur le choix de ru}

La compilation des résultats d'auscultation a montré de fortes analogies dans la réponse des pressions générées dans les ouvrages de certaines régions, comme le montre la figure 7 (BINQUET, 1986) qui représente l'évolution du coefficient ru en fonction de la hauteur de quatre barrages tunisiens. Cette similitude est alors synthétisée sous la forme d'un coefficient ru, représentatif d'une zone géographique, d'un certain type de sol et de dispositifs constructifs courants. Très pratique, cette méthode demande cependant une forte expé rience pour être employée. Tout écart aux conditions

Tableau 2. - Calcul de la pression d'eau de la cellule B6 à Mirgenbach.

Table 2. - Computation of the water pressure of the gauge B6-Mirgenbach Dam.

\begin{tabular}{|c|c|c|c|r|}
\hline$\sigma_{v}(\mathrm{kPa})$ & $\mathrm{Sr}(\%)$ & $\mathrm{P}_{\mathrm{a}}(\mathrm{kPa})$ & $\mathrm{P}_{\mathrm{w}}-\mathrm{P}_{\mathrm{a}}(\mathrm{kPa})$ & $\mathrm{P}_{\mathrm{w}}(\mathrm{kPa})$ \\
\hline 23 & 95 & 3,5 & -56 & $-52,5$ \\
155 & 96 & 20,5 & -49 & $-28,5$ \\
309 & 97 & 52,0 & -40 & 11,5 \\
\end{tabular}


moyennes peut être préjudiciable. Par exemple, les résultats de Binquet sont relatifs à des teneurs en eau proches de l'optimum, comprises entre $W_{\mathrm{OPN}}-1$ et $W_{\text {OPN }}+2$ et des vitesses de construction comprises entre 10 et $30 \mathrm{~cm} /$ jour.

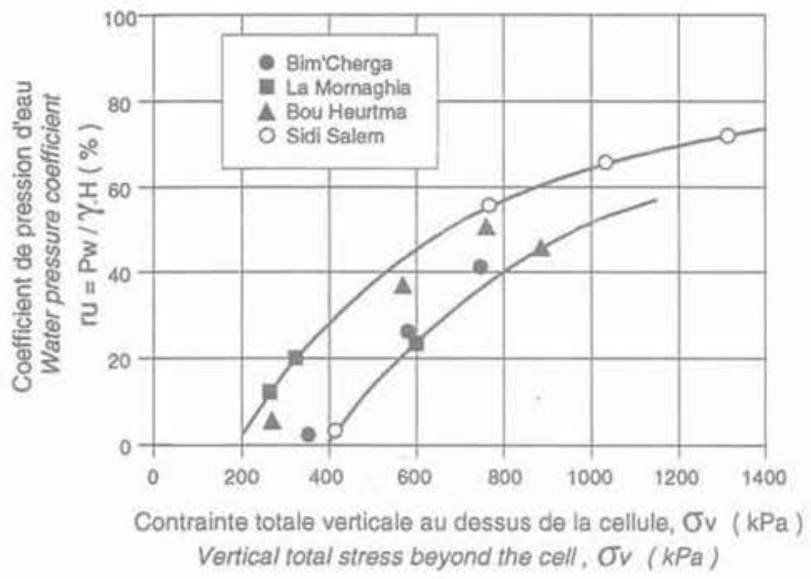

Fig. 7. - Evolution de ru sur quatre barrages de Tunisie, d'après BINQUET (1986).

Fig. 7. - ru evolution on four Tunisie Dams. (BINQUET, 1986).

\subsection{Méthode basée sur la comparaison des essais drainés et non drainés}

Par extrapolation du postulat de Terzaghi aux sols proches de la saturation, la compressibilité ne dépend que de la contrainte effective. Aussi, un essai œedométrique non drainé donne l'indice des vides en fonction de la contrainte totale, tandis qu'un essai drainé le donne en fonction de la contrainte effective, la différence entre les deux donne la pression interstitielle. Cette méthode astucieuse, suggérée par Hilf est particulièrement adaptée aux sites éloignés, où seul un laboratoire peu équipé est disponible.

\subsection{Prise en compte de la consolidation}

L'application des méthodes précédentes aboutit régulièrement à des pressions plus élevées que la réalité. Cela est attribué au fait que les pressions se sont en partie dissipées. BISHOP et al. (1964), qui avait noté ce décalage, proposa d'ajouter la consolidation à l'approche précédente. Le calcul consiste à cumuler les incréments de pression déterminés à chaque pas de temps. Chaque incrément est la somme d'une montée en pression, calculée par la méthode de Hilf et d'une dissipation, calculée avec la théorie de la consolidation de Terzaghi. $\mathrm{Si}$ le degré de saturation était initialement supposé constant, l'apport des calculs transitoires permet d'évaluer son évolution.

Cette dernière solution fut appliquée à l'analyse de l'arrêt saisonnier du remblai de Mirgenbach. La question consiste à savoir d'où vient l'eau qui monte en pression l'été suivant. Est-ce la pénétration des eaux météorologiques pendant l'arrêt hivernal de 1981-1982 ou un excès d'eau à la mise en place en septembre 1981?
Le calcul intègre la génération de pression déduite du degré de saturation pour l'incrément de contrainte considéré et l'intervalle de temps étudié, calcule la dis. sipation et en déduit le degré de saturation pour une nouvelle étape. Il apparaît que la pénétration des eaux nivales et pluviales atteint au maximum $1 \mathrm{~m}$ (fig. 8). Elle n'a donc pas contribué à déconsolider la zone cisaillée, excepté en sortie de talus. Par contre une couche humide ne suffit pas à expliquer les fortes pressions d'eau qui se consolideraient trop vite. Trois couches humides successives sont nécessaires pour expliquer le glisse. ment.

\section{UNE APPROCHE TRIPHASIQUE COUPLÉE : UDAM}

\subsection{Les approches couplées existantes}

Les calculs plus complets, prenant en compte les phénomènes de consolidation avec la méthode des élé. ments finis sont apparus à la fin des années soixantedix (CHANG et DUNCAN, 1977). En France, elle a été développée pour les sols saturés au LCPC par J.P. MA. GNAN, au LMSS de l'Ecole centrale de Paris par D. AUBRY et au CERMES par B. GATMIRI.

Les premières approches concernant les sols non saturés, modélisent les sols proches de la saturation, compactés côté humide. Une première solution consiste à considérer le mélange eau-air comme un fluide compressible (GHABOUSSI and KWANG, 1982). Une telle approche est satisfaisante dans le cas où l'air est piégé sous forme de bulles occluses. Une autre approche, basée sur la notion de contrainte effective élargie aux sols non saturés de Bishop a également été développée (AUBRY et OZANAM, 1988).

Afin de considérer également les degrés de saturation plus faibles, pour lesquels la phase gazeuse est continue et le concept de contrainte effective de Bishop plus valide, il a paru intéressant de considérer certains concepts de la mécanique des sols non saturés, basés sur l'approche en variables indépendantes (contrainte $\sigma-\mathrm{P}_{0}$, succion $\mathrm{P}_{-}-\mathrm{P}_{w}$ ) et de les appliquer au calcul des barrages en remblai, suivant une approche initialement développée par ALONSO et al. (1988).

\subsection{Influence de la succion sur les propriétés du sol}

Dans les conditions de degré de saturation croissant, la notion de surface d'état (fig. 9), développée par MATYAS et RADHAKRISHNAN (1968), permet de donner les valeurs de l'indice des vides e et du degré de saturation $\mathrm{S}_{f}$ en fonction uniquement des valeurs de la contrainte $\left(\sigma-\mathrm{P}_{\mathrm{f}}\right)$ et de la succion $\left(\mathrm{P}_{\alpha}-\mathrm{P}_{w}\right)$. Des résultats expérimentaux ont en effet montré que partant d'un même point et en suivant des chemins de resaturation soit par augmentation de la contrainte, soit par diminution de la succion, on décrivait une surface appelée surface d'état; en d'autres termes, un sol compacté d'indice des vides $e_{1}$ et de degré de saturation 


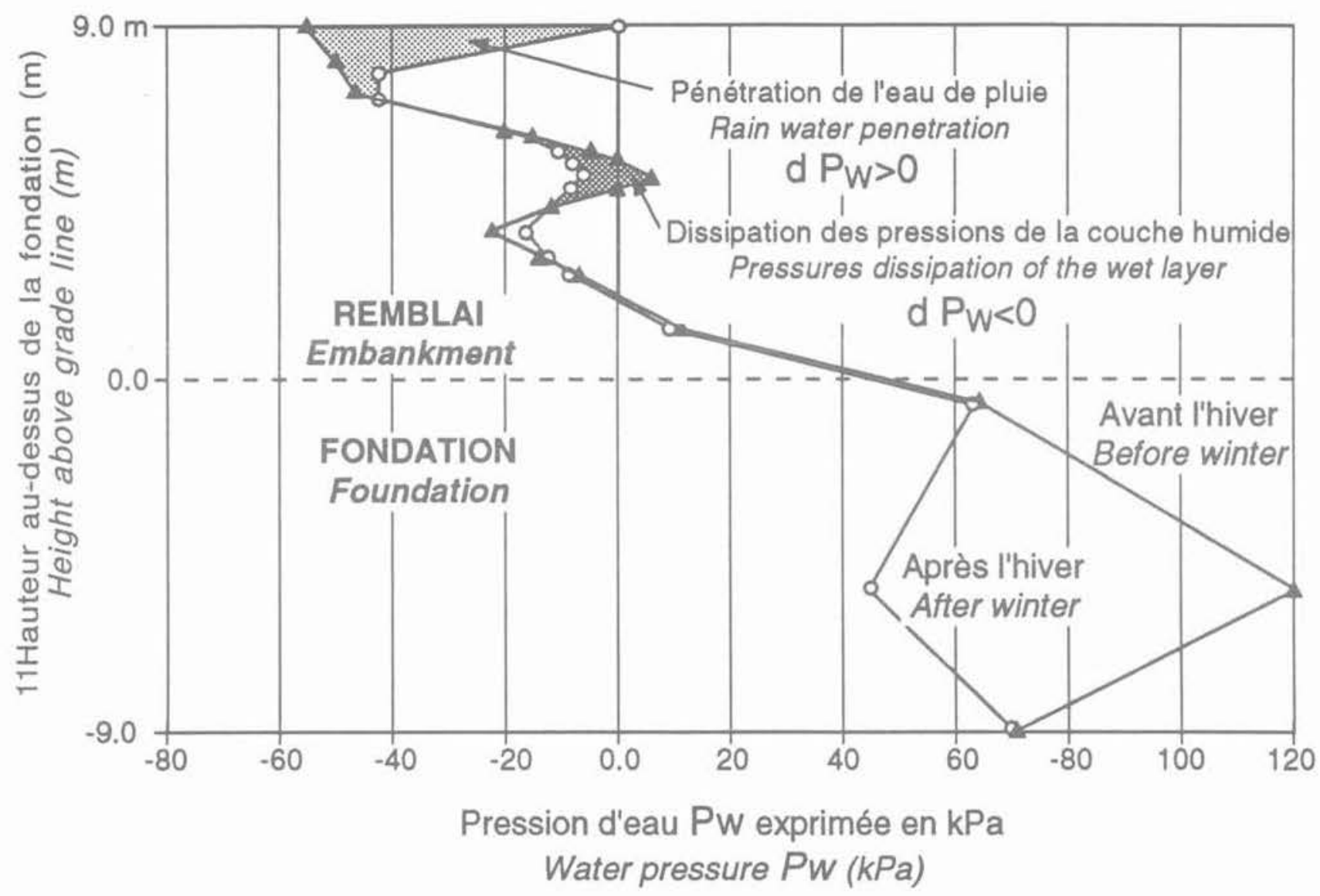

Fig. 8. - Simulation de la génération de pression et de sa dissipation pendant l'hiver 1981-1982 au sein du barrage de Mirgenbach.

Fig. 8. - Simulation of pressure generation and its dissipation during the winter of 81-82 within the Mirgenbach Dam.

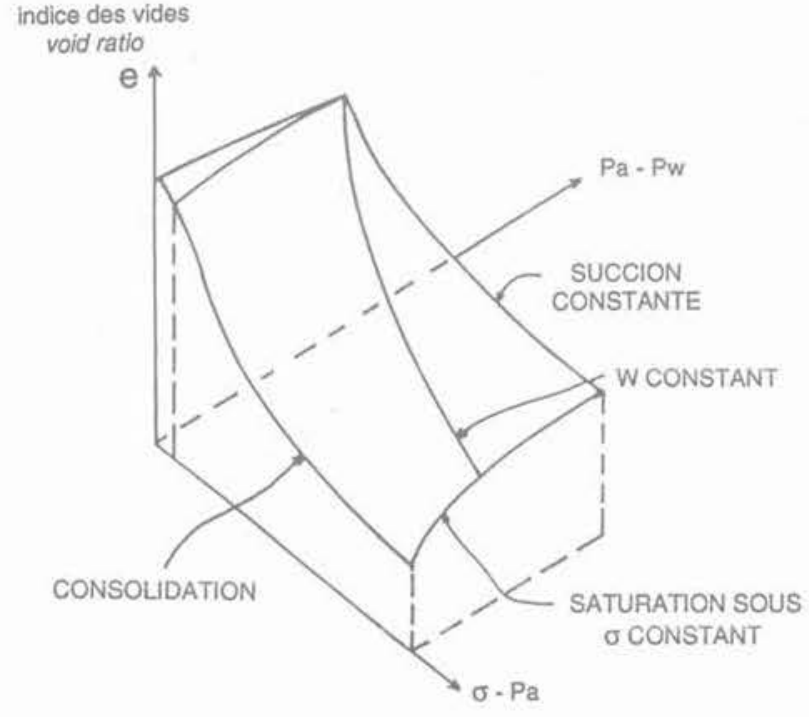

Fig. 9. Compressibilité fonction de deux variables indépendantes.

Fig. 9. - Compressibility with respect to two independant variables.

(MATHYAS et RADHAKRISNA, 1968).
$\mathrm{Sr}_{1}$, soumis à divers chemins de resaturation (compression à teneur en eau constante, humidification ou chargement drainé) atteindra des valeurs finales $e_{2}, \mathrm{Sr}_{2}$, indépendantes du chemin suivi. Cette notion permet de rendre compte de l'augmentation de la compressibilité du matériau compacté avec la diminution de la succion (ou l'augmentation de la teneur en eau). Elle décrit également le tassement au remouillage observé sous contrainte constante, appelé effondrement, et qui est caractéristique du comportement des noyaux de grands barrages lors de la première mise en eau. Un tel phénomène ne peut être expliqué par l'application stricte de la notion de contrainte effective qui prévoit un gonflement suite à laugmentation de la pression d'eau.

L'effet de la succion sur la résistance au cisaillement d'un sol compacté et illustré par les courbes effort-déformation de la figure 10 (DELAGE et al., 1987) obtenues sur un appareil triaxial à succion contrôlée, avec des succions variant entre 50 et $800 \mathrm{kPa}$. Ces résultats montrent la diminution du module et de la résistance du matériau en fonction de la diminution de la succion et donc de l'augmentation de la teneur en eau. Ceci est caractéristique également de l'évolution de l'argile lors de la construction du barrage. Le module, la cohésion sont des fonctions linéaires de la succion et 
l'angle de frottement en est une fonction non linéaire. La borne inférieure est la valeur à saturation.

Il est alors possible de définir un coefficient, dénommé SF (fig. 10), qui est le rapport du déviateur principal sur le déviateur maximal à conditions identiques contrainte et succion identiques). Pour l'ingénieur, ce paramètre est très instructif, car c'est un indice de la sécurité locale et transitoire.

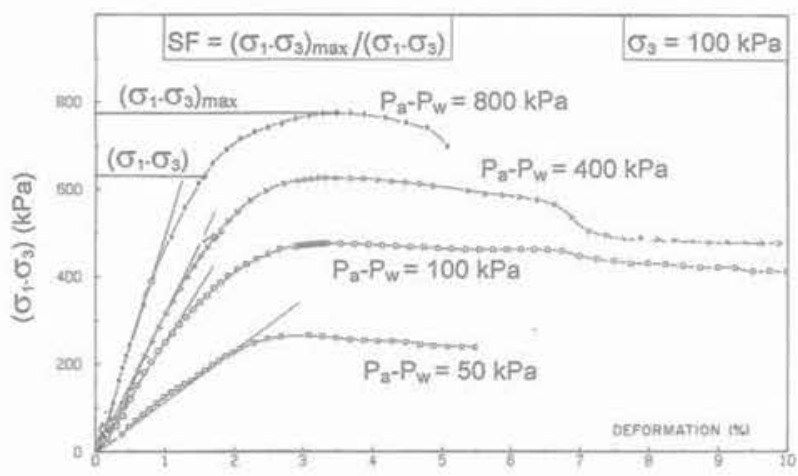

Fig. 10. - Augmentation de la rigidité et de la résistance avec la succion (DELAGE et al. 1987), et définition du coefficient local de sécurité SF.

Fig. 10. - Increase of stiffness and resistance with suction (DELAGE et al. 1987), and definition of the local safety factor SF.

Les transferts d'eau au sein d'un sol non saturé dépendent de l'indice de vides et du degré de saturation. On peut s'interroger sur la représentativité de la forte non linéarité des perméabilités mesurée en labo. ratoire avec le degré de saturation. L'absence de cette relation amène souvent des erreurs de l'ordre de 1 à 4 sur les durées auscultées dans les ouvrages réels.

Les transferts d'air dépendent de l'indice des vides et du degré de saturation à travers l'expression de YOS. HIMI et OSTERBERG (1963).

La loi de HENRI a été appliquée pour traduire la dissolution de l'air dans l'eau.

\subsection{Le logiciel UDAM}

Les notions présentées précédemment concernant cer. tains aspects de l'influence de la succion sur les propriétés mécaniques des sols non saturés ont été intégrés dans le logiciel bidimensionnel UDAM par NANDA (1989). Les propriétés de résistance au cisaillement décrites sur la figure 10 ont été intégrées dans un modèle hyperbolique de type Duncan, en prenant en compte l'effet de ramollissement dû à la succion. Les expres. sions de surface d'état, ainsi que les équations relatives aux transferts d'eau et d'air sont inspirées de ALONSO et al. (1988).

Par rapport aux travaux d'ALONSO et al. (1988), la principale différence réside dans la résolution entière. ment couplée du système. Chaque noud possède quatre degrés de liberté: les déplacements horizontal et vertical, la pression d'air et la pression d'eau. La construction par couches est modélisée par un traite. ment type « matrice fixe » avec tous les degrés de liberté activés au fur et à mesure de la construction. Trois types de matériaux sont considérés: drainé, saturé et non saturé. Les conditions aux limites sont soit débit nul soit pression d'air et pression d'eau fixées par l'utilisateur. Ecrit en fortran, le code peut fonctionner sur microordinateur compatible PC et station de travail.

\subsection{Premiers résultats}

Plusieurs tests ont été réalisés (FRY et al,, 1991). Les résultats apparaissent riches d'enseignement, quelques uns sont rappelés ci-dessous.

La figure 11 représente l'évolution de la pression d'eau et la figure 12 celle du coefficient de sécurité local SF en cours de construction. L'ouvrage étudié est un barrage homogène avec filtre, de $39 \mathrm{~m}$ de haut et de pente $\mathrm{H} / \mathrm{V}=3 / 1$, compacté côté humide. Il apparaît que l'état le plus critique n'est pas la fin de construction. Or. la fin de construction est souvent la seule situation du scénario de dimensionnement envisagé.
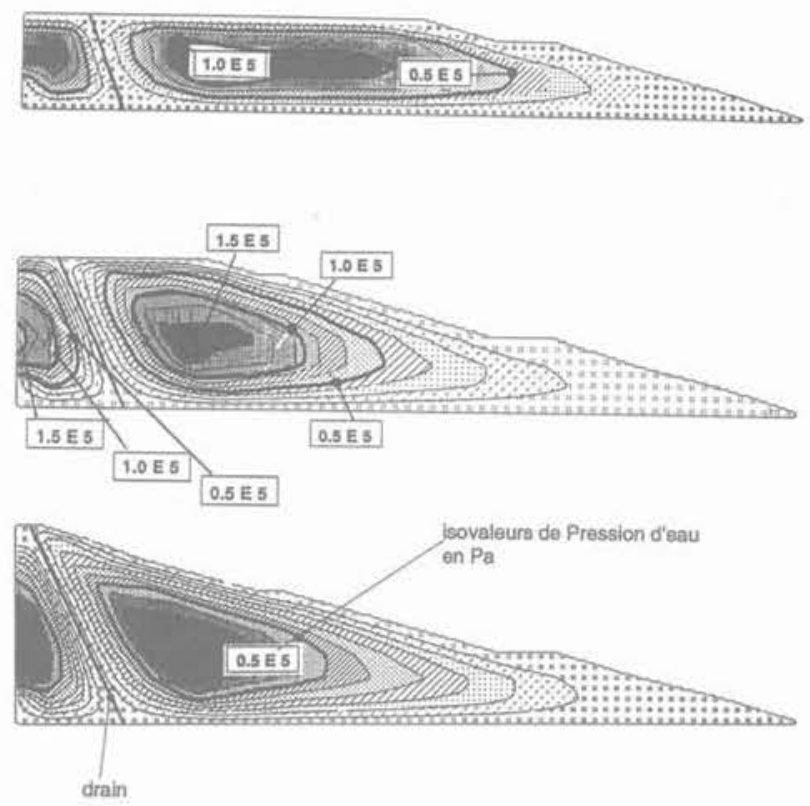

Fig. 11. - Evolution de la pression d'eau simulée par UDAM en cours de construction.

Fig. 11. - Simulation of water pressure contours during construction-UDAM.

La figure 13 met en évidence une localisation des contraintes dans un remblai homogène de $33 \mathrm{~m}$. Cette localisation est mise en évidence par la forme des zones où le coefficient de sécurité local est inférieur à 1,3 . Cela est à rapprocher de la pratique, où il est recommandé de ne pas descendre en dessous d'un coefficient global de 1,3 .

La figure 14 montre un phénomène essentiel : le report de charge obtenu à partir d'une forte teneur en eau initiale et d'une vitesse de construction élevée. Le calcul 

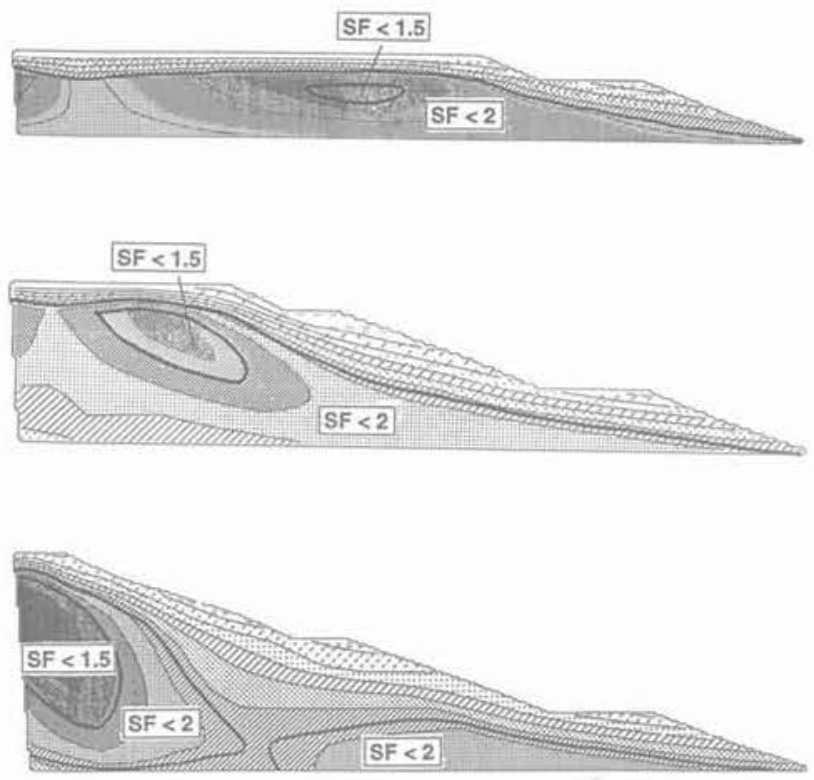

Fig. 12. - Evolution du coefficient de sécurité local simulé par UDAM en cours de construction.

Fig. 12. - Simulation of local safety factor variation during construction-UDAM.

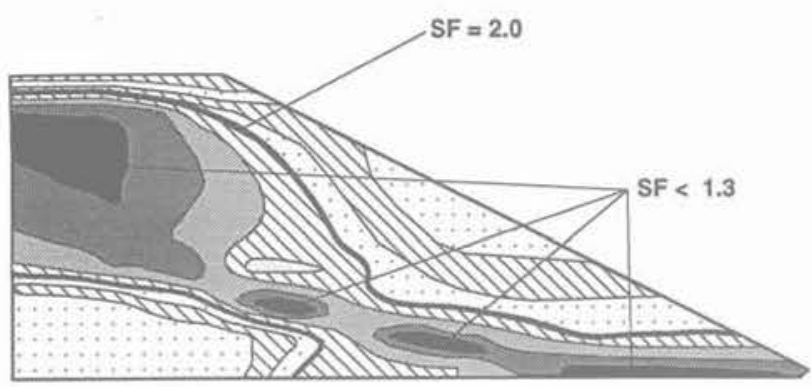

Fig. 13. - Apparition d'une localisation du cisaillement simulée par UDAM et repérée par la répartition du coefficient de sécurité $<1,3$.

Fig. 13. - Simulation of shear band defined by local safety factor < 1.3-UDAM

couplé semble être une voie intéressante pour simuler l'amorce d'une rupture progressive. La génération de pressions, entraîne une ramollissement du sol puis un transfert de charge qui à son tour augmente la génération de pression, etc.

Ces phénomènes suspectés depuis longtemps n'étaient pas jusqu'à présent correctement accessibles au calcul. Aussi importe-t-il maintenant de vérifier la véracité de ces prédictions.

\subsection{Qualification et domaine d'application du modèle}

Ces calculs ont été obtenus avec les paramètres du limon de Jossigny compacté à $w_{\mathrm{opN}}+2 \%$.
Le calcul semble reproduire qualitativement le type de rupture de Mirgenbach. Pour le prouver, il importe d'avoir les paramètres non saturés de cette argile. Cela permettrait de qualifier la démarche.

D'après la terminologie proposée par TARDIEU et OZANAM (1991) et acceptée par la Commission internationale des Grands Barrages, la qualification comprend deux parties:

- la validation: vérification du bon fonctionnement des algorithmes et des procédures informatiques ;

- la justification : vérification des hypothèses physiques du modèle et du choix des parametres de calcul.

Ces deux parties ont été abordées initialement dans le cadre du GRECO-Géomatériaux. Maintenant elles font partie d'un programme de travail collectif CERMES-MECASOL-EDF soutenu par le ministère de la Recherche. Il s'agit de vérifier la pertinence du choix et du pas de temps d'une part et d'autre part de justifier le programme en retrouvant des pathologies connues (Mirgenbach) ou des comportements d'ouvrages auscultés, à partir de procédures expérimentales simples et peu coûteuses de détermination des surfaces d'état. En effet cette approche ne sera appliquée que si la détermination expérimentale est simple et industrielle.

L'avantage de cette modélisation est sa rapidité. Les limites sont celles du modèle: le domaine d'application est à priori uniquement la construction et la mise en eau. La vidange est théoriquement déconseillée, mais l'erreur est d'autant plus faible que la vidange est rapide. Les corrélations de FLEUREAU (1989) seront utiles et les mesures in situ seront indispensables pour le choix des paramètres qui restera en dernier ressort du do. maine de l'expert.

\section{CONCLUSIONS}

L'étude des sols compactés était jusqu'à présent menée avec les concepts de la mécanique des sols saturés. Ainsi, la stabilité des barrages en terre est vérifiée par le calcul à la rupture, la méthode des tranches, dans lequel les pressions d'eau sont estimées de manière empirique. Maintenant le développement de la mécanique des sols non saturés est suffisamment avancé pour mettre à la disposition de l'ingénieur des outils qui prennent en compte l'état initial non saturé et son impact sur la génération de pression et la stabilité. Le code UDAM développé par le CERMES et l'EDF est parmi les premiers de ces outils. Il prend en compte l'action de la pression capillaire sur les propriétés du sol et les transferts d'eau et d'air qui sont résolus de manière couplée. Les applications en non saturé sont nom breuses. Il est possible de mettre en évidence les effets des dispositifs constructifs, drains, vitesse de construction, choix de la teneur en eau, sur la sécurité de l'ouvrage, $\mathrm{Ce}$ code est en cours de qualification. Si les verifications sur ouvrage réel s'avèrent toutes correctes, il devrait rendre de rapides services à l'ingénieur qui projette les spécifications et les dispositifs de construction d'un barrage en terres et en enrochements. 


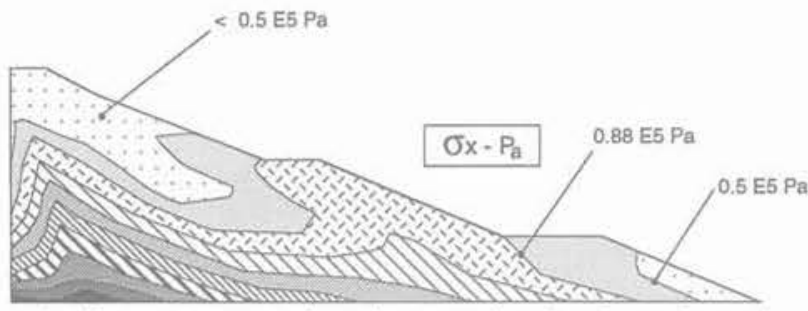

a): Forte concentration de contrainte horizontale sur le tiers inférieur du parement aval.

High concentration of horizontal stress on the lower third of dowstream facing.

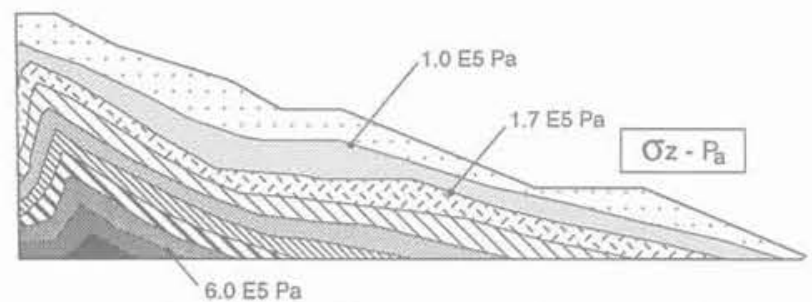

b) : Concentration de contrainte verticale Concentration of vertical stress.

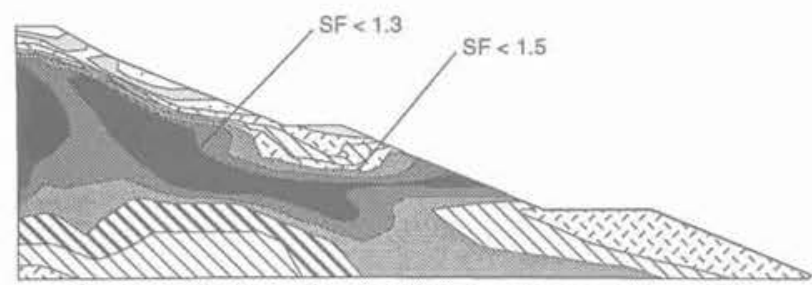

c) : Localisation de coefficients de sécurité $<1,3$. Localisation of safety factors $<1.3$

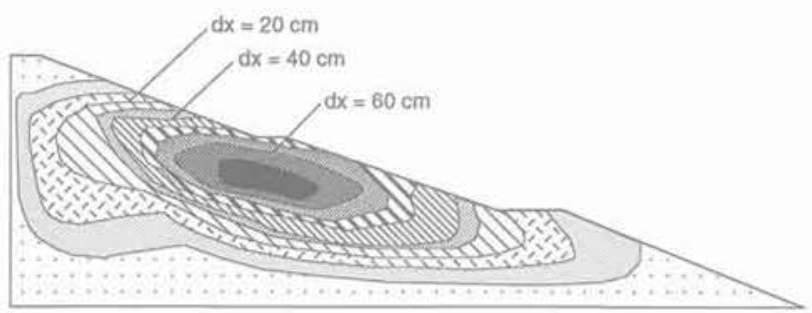

d) : Concentration des déplacements horizontaux. Concentration of horizontal displacements.

Fig. 14. - Existence et impact d'un report de charge simulé par UDAM.

Fig. 14 - Simulation of stress redistribution- UDAM.

\section{BIBLIOGRAPHIE}

ALONSO A.E., GENS A. and HIGHT A. (1987), Special problem soils. General report. IX ECSMFE, Dublin, vol. 3, pp. 1087-1147.

ALONSO A.E., BATTLE F., GENS A. and LLORET A. (1988). Consolidation analysis of partially saturated soils. Application to earthdam construction. International Conference on Numerical Methods in Geotechnics, Innsbruck, vol. 3, pp. 1303-1308.
AUBRY D. and OZANAM O., Free-surface tracking through non-saturated models. International Conférence on Numerical Methods in Geomecha. nics, Innsbruck, vol. 2, pp. 757-763.

BINQUET J. (1986), Comportement en cours de construction des remblais argileux des barrages de Birm'Cherga, Lamonarghia, Sidi Saleh et Bou Heurta. Formation continue EDF, Barrages en argile, $14-15$ mai 1986.

BISHOP A.W. (1954), The use of pore pressure coefficients in practice. Géotechnique, vol. IV, pp. 143. 148.

BISHOP A.W. (1957), Some factors controlling the pore pressure set up during the construction of earth dams. Proc. 4th International Conference on Soil Mechanics and Foundation Engineering, Londres, vol. II, 294-300.

BISHOP A.W., HENKEL D.J. (1962), The measurement of soil properties in the triaxial test, $2^{\circ}$ édition, Edward Arnold Publisher LTD.

BISHOP A.W., KENNARD M.F., VAUGHAN P.R. (1964), Developments in the measurement and interpretation of pore pressure in earth dams. 8th International Congress On Large Dams, Edinburgh R4, Q29, pp. 47-70.

CHANG C.S., DUNCAN J.M. (1977), Analysis of consolidation of earth and rockfill dams. Report $n^{\circ} \mathrm{TE}$ 77.3, dept. of Civil Engineering, University of California, Berkeley.

DELAGE P. SURAJ DE SILVA G.P.R. and DE LAURE E. (1987). A new triaxial apparatus for non saturated soils. IXth ECSMFE Dublin, vol. 1, pp. 25-28.

FLEUREAU J.M. (1989), Rapport d'activité du LMSS de l'Ecole Centrale de Paris, GRECO-GEOMATERIAUX, Rapport scientifique 1989, pp. 202 . 210.

FRY J.J., DELAGE P., NANDA A., GATMIRI B. et ALONSO E.E. (1991), Approche triphasique de la construction des remblais argileux. $17^{\circ}$ Congrès de la Commission International des Grands Barrages, Vienne, juin 1991, vol. V, pp. 538-543.

GHABOUSSI J., KWANG J.K. (1982), Analysis of saturated and partially saturated soils. International Symposium on Numerical Models in Geomecha. nics, Zurich, pp. 377-390.

HILF J.W. (1948) Estimating construction pore pressure in rolled earth dams. Proc. 2nd International Conference on Soil Mechanics and Foundation Engineering, Rotterdam, vol. 3, pp. 234-240.

LAMBE T.W. (1961), Residual pore pressures in compacted clay. Proc. 5th International Conference on Soil Mechanics and Foundation Engineering, vol. I/35, pp. 207-211.

LLORET A.. ALONSO E.E. (1980), Consolidation of unsaturated soils including swelling and collapse behaviour. Géotechnique 30, n 4, pp. 449-177.

MATYAS E.L., RADHAKRISHNA A.S. (1968), Volume change characteristics of partially saturated soils. Géotechnique 18, pp. 432-448.

NANDA A. (1989), Analysis of consolidation of embankment dams during construction. Rapport CERMES-EDF, $81 \mathrm{p}$. 
PELLS P.J.N. (1973), Stress ratio effects on construction pore pressures. Proc. 8th International Conference on Soil Mechanics and Foundation Engineering, Moscow, pp. 327-331.

SKEMPTON A.W. (1954), The pore pressure coefficients A and B. Géotechnique, vol. IV, pp. 143 . 148 .
TARDIEU B. et OZANAM O. (1991), Validation et jus tification. Comptes rendus des Journées scientifiques du GRECO-Géomatériaux, Aussois, pp. 827. 831.

YOSHIMI Y., OSTERBERG J.O. (1963), Compression of partially saturated cohesive soils. Journal of Soil Mechanics and Foundation Division, ASCE SM4, pp. $1-24$. 